\title{
The challenging diagnosis and follow-up of skull base osteomyelitis in clinical practice
}

\author{
Alice B. Auinger ${ }^{1} \cdot$ Valerie Dahm $^{1} \cdot$ Isabella Stanisz ${ }^{1}$. Ursula Schwarz-Nemec ${ }^{2} \cdot$ Christoph Arnoldner $^{1}$ (I)
}

Received: 30 August 2020 / Accepted: 15 December 2020 / Published online: 28 January 2021

(c) The Author(s) 2021

\begin{abstract}
Purpose The disease activity of skull base osteomyelitis can be challenging to assess by means of conventional imaging methods and renders monitoring of the disease difficult, especially in areas with restricted access to nuclear medicine imaging. Here, we provide clinically relevant data on the management of skull base osteomyelitis including assessment, treatment, and follow-up strategies with regards to the role of imaging.

Method A chart review was performed including 30 patients treated for SBO from 1993 to 2015. Clinical findings, treatment procedures, and complication rates were assessed. Special attention was paid to imaging procedures.

Results The overall mortality rate was $36.7 \%$ and increased to $45 \%$ when cranial nerve palsies were present. An initial computed tomography (CT) scan was performed in all patients, MRI in $60 \%$ and nuclear imaging in $33 \%$. CT scans failed to detect progression or regression in up to $80 \%$ after four to nine months. MRI examinations could reveal changes at a higher rate compared to $\mathrm{CT}$. Nuclear medicine functional imaging was most likely to assess disease activity.

Conclusion A combination of different imaging modalities is recommended for diagnosing SBO. For the follow-up, MRI is preferable to $\mathrm{CT}$ as changes can be detected more readily with MRI. If available, nuclear medicine imaging should guide the decision of treatment discontinuation.
\end{abstract}

Keywords Skull base osteomyelitis $\cdot$ Osteomyelitis $\cdot$ Malignant otitis externa $\cdot$ Necrotizing otitis externa $\cdot$ Cranial nerve palsy

\section{Introduction}

Skull base osteomyelitis (SBO) is a rare but severe and lifethreatening disease. It mainly occurs secondary to otogenic and sinugenic infections via contiguous spread. Pain is often the only symptom and typical clinical findings associated with external otitis or otitis media are often missing [1]. Diabetes, cardiovascular diseases, and immunosuppression are known to be the most common comorbidities in SBO patients [2-4]. Despite improved diagnostic modalities and the use of modern antibiotic agents, mortality and morbidity rates remain high. Data of the Californian population

Christoph Arnoldner

christoph.arnoldner@meduniwien.ac.at

1 Department of Otorhinolaryngology, Medical University of Vienna, Waehringer Guertel 18-20, 1090 Vienna, Austria

2 Department of Biomedical Imaging and Image-Guided Therapy, Medical University of Vienna, Waehringer Guertel 18-20, 1090 Vienna, Austria revealed a median incidence of otogenic SBO of 75.5 cases per year [5]. Due to an aging and subsequently increasingly multimorbid population, SBO is on the rise [6]. Therefore, it is important to raise awareness for this disease as limited evidence of diagnostic, therapeutic, and follow-up strategies render management difficult [7].

Finding the correct diagnosis is the first challenge when faced with the question of SBO. No single imaging modality is able to detect the extent of this disease. Active inflammation is difficult to distinguish from resolving abnormalities in conventional imaging modalities. Nuclear medicine functional imaging methods alone or a combination with high-resolution imaging for anatomy such as single-photon emission computed tomography (SPECT) or positron emission tomography (PET) with computed tomography (CT) or magnetic resonance imaging (MRI) are recommended but not often available [8-10].

Once the diagnosis of SBO is made, the next difficulty is finding the correct therapy and consecutively deciding on the necessary duration of treatment. Pseudomonas 
aeruginosa has been reported to be the main pathogen in $50-98 \%$ of SBO $[3,11,12]$. However, in up to a third of patients no pathogen can be isolated [13, 14]. Most patients are treated with topical or oral antibiotics when they present at primary care physicians. In cases where recovery has failed, pre-treatment can lead to negative cultures. Surgery was once advocated as the treatment method of choice [15]. Long-term intravenously administered antibiotic agents are indicated when the diagnosis is made with a median of 3.5 agents used in a patient $[2,16]$. Hyperbaric oxygen therapy might be an effective adjuvant in advanced or treatmentrefractory SBO [17].

Further, there is no consensus on the preferred imaging modality to assess disease progress or relapse as well as treatment efficacy. Primary or secondary care hospitals do not have the ability to assess disease activity with nuclear medicine imaging. Most clinicians have to rely on conventional imaging modalities such as MRI and CT. In this study, patients with clinical signs of SBO, who were treated in a tertiary care hospital, were evaluated. Apart from outcome parameters such as cranial nerve function and mortality rates, special attention was paid to conventional follow-up imaging. The main aim of the present study was to evaluate the outcome of patients with SBO and to assess the best possibility for conventional follow-up imaging methods.

\section{Materials and methods}

A chart review was performed including patients with clinically diagnosed SBO, who were treated from January 1993 to December 2015 in a tertiary care hospital. The patients' database was searched for the diagnosis "malignant otitis externa", "skull base osteomyelitis", and "petrositis". A total of 30 patients met inclusion criteria, which were clinical signs of SBO such as inflammation of the ear and/or facial nerve palsy and/or erosion of the temporal bone on any imaging method. An otogenic cause was assumed in $80 \%(n=24)$, a sinugenic cause in 3.3\% $(n=1)$ and in $16.7 \%$ $(n=5)$ the origin of the infection remained unclear.

Based on medical records, hospitalization time, clinical symptoms including complications such as cranial nerve palsies, comorbidities, culture results of ear swabs, treatment, and the mortality rate were assessed. If available, histopathologic reports of biopsies and laboratory results including leucocyte count, creatinine clearance, and C-reactive protein (CRP) on admission and discharge were evaluated.

The role of high-resolution CT in diagnosing SBO and during follow-up procedures has been a matter of discussion. Therefore, special attention was paid to imaging reports performed for diagnosis and during follow-up of SBO. Imaging modalities included CT, MRI, and nuclear medicine functional imaging methods. Nuclear medicine imaging methods such as scintigraphy, single-photon emission tomography (SPECT) or positron emission tomography (PET) were performed to assess functional aspects rather than anatomical structures. Tc-99m MDP detects osteoblastic activity and was the most frequently used tracer in this study. Other tracers used in the current study were Tc-99m labelled leukocyte antibodies, which accumulate in infectious areas such as found in SBO and beta-emitting tracers like 2-Fluor-2-Desoxy-Glucose (FDG), which detect increased metabolism as in infectious or malignant diseases. Four CT scans were performed for CT-guided biopsy and abscess drainage and were not included in the analysis.

During hospitalization, all patients were primarily treated with broad-spectrum intravenous antibiotics until sensitivity patterns of culture results were available. Blood glucose levels were optimized if necessary. Cases which could not be connected to SBO where classified as death due to other reasons. The study was approved by the local ethics committee (EK 1286/2016). Due to the retrospective nature of this study, no informed consent of included patients was required.

\section{Results}

\section{Demographic results and hospitalization time}

Of 30 included patients (10 females, 20 males), 50\% ( $n=15)$ were referred from previous in-patient treatment at other hospitals, and 50\% $(n=15)$ were referred from general practitioners. The mean age on admission was $73.1 \pm 13.5$ years. The right temporal bone was affected by $36.7 \%(n=11)$ and the left side in $63.3 \%(n=19)$. A second admission was necessary for $33.3 \%(n=10)$. Some patients required inpatient treatment for a third or fourth time (see Table 1 for demographic and clinical characteristics of all included patients). Patients were admitted $1.4 \pm 0.7$ times on average with a mean hospitalization time of $50.4 \pm 53.1$ days.

\section{Clinical findings, symptoms and comorbidities}

Clinical symptoms and findings on admission were distributed as follows (multiple symptoms per patient are listed): otalgia and headache $76.6 \%(n=23)$ followed by otorrhea $40.0 \%(n=12)$, inflammation of the external ear canal $36.7 \%$, $(n=11)$, granulation tissue of the external ear canal $26.7 \%(n=8)$, and perforation of the tympanic membrane $6.7 \%(n=2)$.

The most common comorbidity was diabetes $(73.3 \%$, $n=22)$. Arterial hypertension was observed in $46.7 \%$ $(n=14)$, peripheral vascular disease in $20.0 \%(n=6)$, and $26.7 \%(n=8)$ of included patients were immunosuppressed due to kidney transplant, lymphoma or polyarthritis. 
Table 1 Demographic details of included subjects

\begin{tabular}{|c|c|c|c|c|c|c|c|c|}
\hline ID & Sex & Age & $\mathrm{CNP}$ & Side & HT & Admissions & Survival & Comorbidities \\
\hline 1 & M & 71 & $\mathrm{n}$ & $\mathrm{r}$ & 102 & 3 & $\mathrm{Y}$ & $\mathrm{DM}, \mathrm{H}$ \\
\hline 2 & $\mathrm{~F}$ & 70 & VII & 1 & 80 & 2 & $\mathrm{~N}$ & $\mathrm{DM}$ \\
\hline 3 & M & 36 & $\mathrm{n}$ & 1 & 20 & 1 & $\mathrm{Y}$ & $\mathrm{n}$ \\
\hline 4 & M & 87 & $\mathrm{n}$ & $\mathrm{r}$ & 38 & 2 & $\mathrm{~N}^{\mathrm{a}}$ & $\mathrm{H}$ \\
\hline 5 & M & 86 & VII & 1 & 233 & 4 & $\mathrm{~N}$ & DM, CKI \\
\hline 6 & M & 86 & VII & 1 & 30 & 2 & $\mathrm{Y}$ & $\mathrm{n}$ \\
\hline 7 & M & 51 & $\mathrm{n}$ & 1 & 45 & 1 & $\mathrm{~N}^{\mathrm{a}}$ & DM, PAVK \\
\hline 8 & M & 83 & $\mathrm{n}$ & 1 & 29 & 1 & $\mathrm{~N}$ & $\mathrm{DM}$ \\
\hline 9 & M & 68 & $\mathrm{n}$ & 1 & 15 & 1 & $\mathrm{Y}$ & $\mathrm{DM}$ \\
\hline 10 & $\mathrm{~F}$ & 79 & VII & 1 & 34 & 1 & $\mathrm{~N}$ & $\mathrm{DM}, \mathrm{H}$ \\
\hline 11 & $\mathrm{~F}$ & 79 & III, VI, V, VII & 1 & 145 & 1 & $\mathrm{~N}$ & $\mathrm{H}$, Polyarthritis \\
\hline 12 & $\mathrm{~F}$ & 79 & VII & 1 & 17 & 1 & $\mathrm{~N}$ & DM, H, CAVK \\
\hline 13 & M & 85 & VI & 1 & 7 & 1 & $\mathrm{~N}$ & DM, CAVK \\
\hline 14 & M & 87 & $\mathrm{n}$ & $\mathrm{r}$ & 9 & 1 & $\mathrm{Y}$ & DM, H, CAVK \\
\hline 15 & M & 63 & VII & $\mathrm{r}$ & 6 & 1 & $\mathrm{~N}^{\mathrm{a}}$ & DM, H, CAVK, KT \\
\hline 16 & M & 72 & VII & 1 & 19 & 1 & $\mathrm{~N}$ & $\mathrm{H}, \mathrm{KT}$ \\
\hline $17^{\dagger}$ & M & 81 & II, VII, IX, X & 1 & - & - & $\mathrm{N}$ & $\mathrm{DM}, \mathrm{H}, \mathrm{cAVK}$ \\
\hline 18 & $\mathrm{~F}$ & 58 & VII, X, XI & $\mathrm{r}$ & 107 & 2 & $\mathrm{~N}$ & $\mathrm{H}, \mathrm{LY}$ \\
\hline 19 & $\mathrm{~F}$ & 77 & $\mathrm{n}$ & $\mathrm{r}$ & 50 & 1 & $\mathrm{Y}$ & $\mathrm{DM}$ \\
\hline 20 & M & 71 & VII & 1 & 17 & 1 & $\mathrm{Y}$ & $\mathrm{DM}, \mathrm{H}$ \\
\hline 21 & M & 93 & VI & 1 & 44 & 2 & $\mathrm{Y}$ & cAVK \\
\hline 22 & $\mathrm{~F}$ & 59 & VII & $\mathrm{r}$ & 19 & 1 & $\mathrm{Y}$ & DM, cAVK \\
\hline 23 & M & 60 & VII, IX, XII & 1 & 25 & 1 & $\mathrm{Y}$ & DM, H \\
\hline 24 & $\mathrm{~F}$ & 88 & $\mathrm{n}$ & $\mathrm{r}$ & 16 & 1 & $\mathrm{~N}^{\mathrm{b}}$ & DM, H \\
\hline 25 & M & 70 & VII & $\mathrm{r}$ & 111 & 2 & $\mathrm{Y}$ & DM, cAVK \\
\hline 26 & $\mathrm{~F}$ & 77 & $\mathrm{n}$ & $\mathrm{r}$ & 3 & 1 & $\mathrm{~N}$ & LY \\
\hline 27 & $\mathrm{~F}$ & 72 & VIII & 1 & 156 & 1 & $\mathrm{Y}$ & DM, LY \\
\hline 28 & M & 89 & V, VII, IX, XII & $\mathrm{r}$ & 62 & 1 & $\mathrm{Y}$ & $\mathrm{DM}$ \\
\hline 29 & M & 63 & VI, X, XII & 1 & 28 & 2 & $\mathrm{~N}^{\mathrm{a}}$ & $\mathrm{DM}$ \\
\hline 30 & M & 49 & VI & 1 & 44 & 1 & $\mathrm{Y}$ & $\mathrm{DM}, \mathrm{H}$ \\
\hline
\end{tabular}

†Patient No. 17 was hospitalized at another hospital but second opinion was requested-complete data were not available; $m$ male, $f$ female, $r$ right, $l$ left, $H T$ hospitalization time in days, $y$ yes, $n$ no, CKI chronic kidney disease, $D M$ diabetes mellitus, $H$ arterial hypertension, $K T$ kidney transplantation, $L Y$ lymphoma, $P A D / C A D$ peripheral arterial/coronary artery disease, $N^{\mathrm{a}}$ death not associated with $\mathrm{SBO}, N^{\mathrm{b}}$ reason for death unknown, Admissions number of admissions for inpatient treatment

\section{Diagnostic results}

\section{Laboratory results}

Laboratory results of the following parameters on admission were assessed: leukocyte count of $8.3 \pm 3.0$, CRP of $4.8 \pm 5.9$, and creatinine of $1.2 \pm 0.4$. At patients' discharge, the leukocyte count was $7.5 \pm 3.7$, CRP $1.8 \pm 3.2$, and creatinine $1.8 \pm 0.5$. The glycated hemoglobin $(\mathrm{HbAlc})$ was available from 10 patients and was $8.7 \pm 2.3$.

\section{Microbiologic results}

Microbiologic swabs verified pathogens in $80.0 \%(n=24)$ on the first admission, whereas no pathogen was detected in $20.0 \%(n=6)$ of all patients; multiple pathogens were observed in $36.7 \%(n=11)$ and a single one in $43.3 \%$ $(n=13)$. By far the most frequently detected bacterium was Pseudomonas aeruginosa in $75.0 \%(n=18)$, followed by Staphylococcus aureus in $29.2 \%(n=7)$ and Candida in $8.3 \%(n=2)$. Further pathogens were Corynebacterium $(12.5 \%, n=3)$, Staphylococcus epidermidis and Coagulase-negative Staphylococci in $8.3 \%$ ( $n=2$ each), and Escherichia coli, Streptococcus anginosos, Aspergillus fumigatus/nidulans, Citrobacter freundii, oropharyngeal bacteria, and Propionibacterium acnes in $4.2 \%(n=1$ each). 3-MDRGN Pseudomonas aeruginosa appeared in $8.3 \%(n=2)$. 


\section{Imaging methods}

In total, $70 \mathrm{CT}$ examinations including first and follow-up scans were performed. An initial CT scan was performed in all included patients $(n=30)$. Multiple findings on one scan could be commonly assessed but the most common pathologies were soft tissue changes in 53\% $(n=16)$ and bone erosion in $43 \%(n=11)$. A second CT scan was performed in $70.0 \%(n=21)$ of patients with a mean of $55.5 \pm 52.7$ days between the first and second scan. Disease progression was found in $57.0 \%(n=12)$ of follow-up scans, regression was seen in $14.0 \%(n=3)$ and $29.0 \%$ $(n=6)$ showed no change. A third $(n=10)$, fourth $(n=8)$ and fifth $(n=2)$ CT scan for follow-up was performed in several patients, but most of the scans revealed no change of findings. Details on results are shown in Table 2. CTs performed for nuclear medicine imaging modalities were not included.
An initial MRI scan was performed in $60 \%(n=18)$ of all included patients $43.8 \pm 81.1$ days after the initial CT scan; in two patients, the MRI was performed 7 and 52 days before the CT scan. Multiple findings on one scan could be assessed; the most common findings on the initial scan were bone erosion in 56\% $(n=10)$, nerve erosion in 56\% $(n=10)$, and soft tissue changes in 33\% $(n=6)$. A second MRI scan was performed in $40 \%(n=12)$ of patients with a mean of $82.4 \pm 77.1$ days between the first and second examination. In total, 57 MRI scans were performed on 18 patients including first and follow-up examinations. The main findings, the time between scans and imaging changes are displayed in Table 2. MRIs performed for nuclear medicine imaging modalities were not included.

A sum of an additional 14 nuclear medicine functional imaging examinations were performed in $33.3 \%(n=10)$ of included patients. A positron emission tomography in combination with CT or MRI was performed once in three

Table 2 First and follow-up imaging with CT and MRI

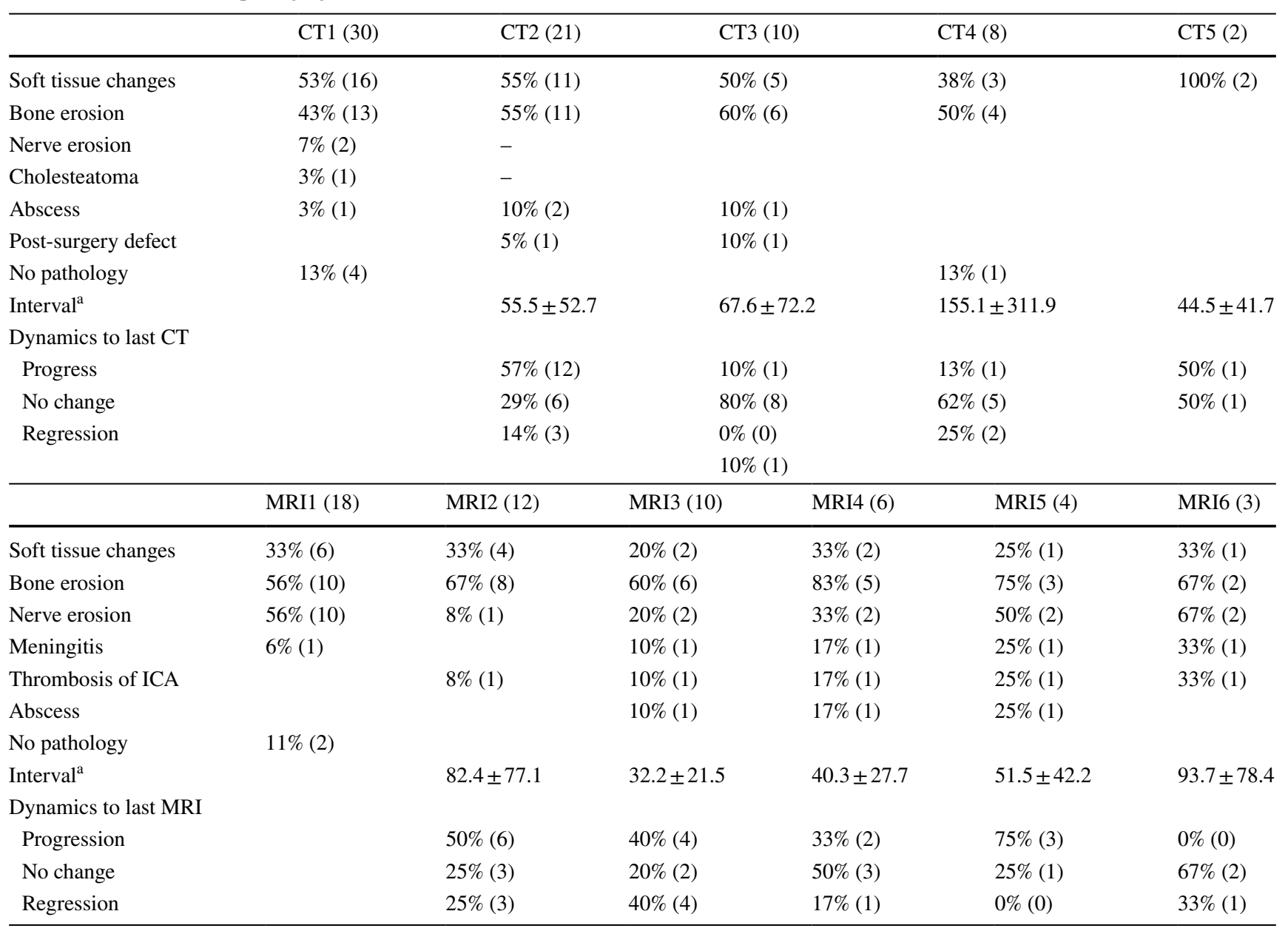

Findings on CT and MRI scans according to reports given as percentages and numbers (brackets). Multiple findings on one scan possible

${ }^{a}$ Time in days between first and second, second and third, third and fourth scan and so forth. CT1 corresponds to the initial CT performed, CT2 to the second, CT3 to the third and so forth. MRI1 corresponds to the initial MRI performed, MRI2 to the second, MRI3 to the third and so forth. The CT and MRI scans of nuclear medicine functional imaging methods are not included 
patients (two PET-CT scans and one PET-MRI), which revealed increased F18-FDG tracer uptake. A scintigraphy was performed 11 times; in eight of these in combination with single-photon emission computed tomography (SPECT), whereof four were performed in combination with a CT (SPECT-CT). Tracer which was used included Tc-99m MDP $(n=5)$, Tc-99m labelled leukocyte antibodies $(n=4)$, and Tc-88m DPD $(n=2)$. All but two scintigraphies revealed increased tracer uptake. One patient without increased tracer uptake showed a clear deterioration on MRI and the patient died in the course of the disease. The tracers used in the latter case were Tc-99m labelled leukocyte antibodies. Another patient without increased uptake of Tc-99m labelled leukocyte antibodies showed increased tracer uptake in the FDG-PET-CT as well as in the scintigraphy with SPECT and Tc-99 $\mathrm{m}$ MDP performed 1 month later.

\section{Treatment}

\section{Conservative treatment}

All patients initially received broad-spectrum intravenous antibiotic therapy until swab results revealed the sensitivity of pathogens. Most commonly used antibiotic agents included B-lactame antibiotics in 56.7\% $(n=17)$, cephalosporines in $40 \%(n=12)$, whereof third-generation cephalosporines were the main administered agent, epoxide antibiotics including fosfomycin in 30\% ( $n=9)$, fluoroquinolones in $23.3 \%(n=7)$, and lincosamides in $23.3 \%(n=7)$. Less frequently used antibiotics included carbapenems in $13.3 \%$ $(n=4)$, fusidic acid, macrolides, glycopeptides, trimethoprim/sulfonamides, lipopeptides, and aminoglycosides in $3.3 \%(n=1)$ each. Patients with suspected fungal causes were treated with triazoles and echinocandines in $3.3 \%$ $(n=1)$ each. Patients received 2.6 different antibiotic agents on average.

\section{Surgery}

On the first admission, surgery was performed in $60.0 \%$ $(n=18)$ of included patients and comprised a mastoidectomy in $27.8 \%(n=5)$, biopsies to rule out malignancy in $33.3 \%$ $(n=6)$, abscess drainage in $22.2 \%(n=4)$, insertion of ventilation tubes in $16.7 \%(n=3)$, one tympanoplasty $(5.6 \%)$, one facial nerve decompression (5.6\%), one functional endoscopic sinus surgery (5.6\%) and removal of necrotic tissue in $16.7 \%(n=3)$ with one skin grafting $(5.6 \%)$. Histological results revealed granulation, necrotic or inflammatory tissue in all specimens. Out of $33.3 \%(n=10)$ readmitted patients, four underwent a second surgery including two mastoidectomies, one VII decompression, and one removal of necrotic tissue with skin grafting.

\section{Mortality rate and complications}

The overall mortality rate was $53.3 \%(n=16)$ of included patients, whereby $36.7 \%(n=11)$ were directly associated with SBO and $16.7 \%(n=5)$ died due to other reasons. Mean time between admission and death was $23.3 \pm 18.7$ weeks. On admission, cranial nerve palsy (CNP) was present in $66.7 \%(n=20)$; single nerve involvement in $70.0 \%(n=14)$ and multiple nerve palsies in $30.0 \%(n=6)$. The most commonly affected nerve was the facial nerve in $75.0 \%(n=15)$. No patient developed a new CNP during treatment. Figure 1 shows the distribution of affected cranial nerves. The mean hospitalization time of patients with CNP including all admissions was $61.3 \pm 49.6$ days. For patients without CNP the mean inpatient treatment duration was $43.6 \pm 24.7$ days.
Fig. 1 Rate of cranial nerve palsies in percentages; roman numerals indicate cranial nerves

\section{Cranial nerve involvement}

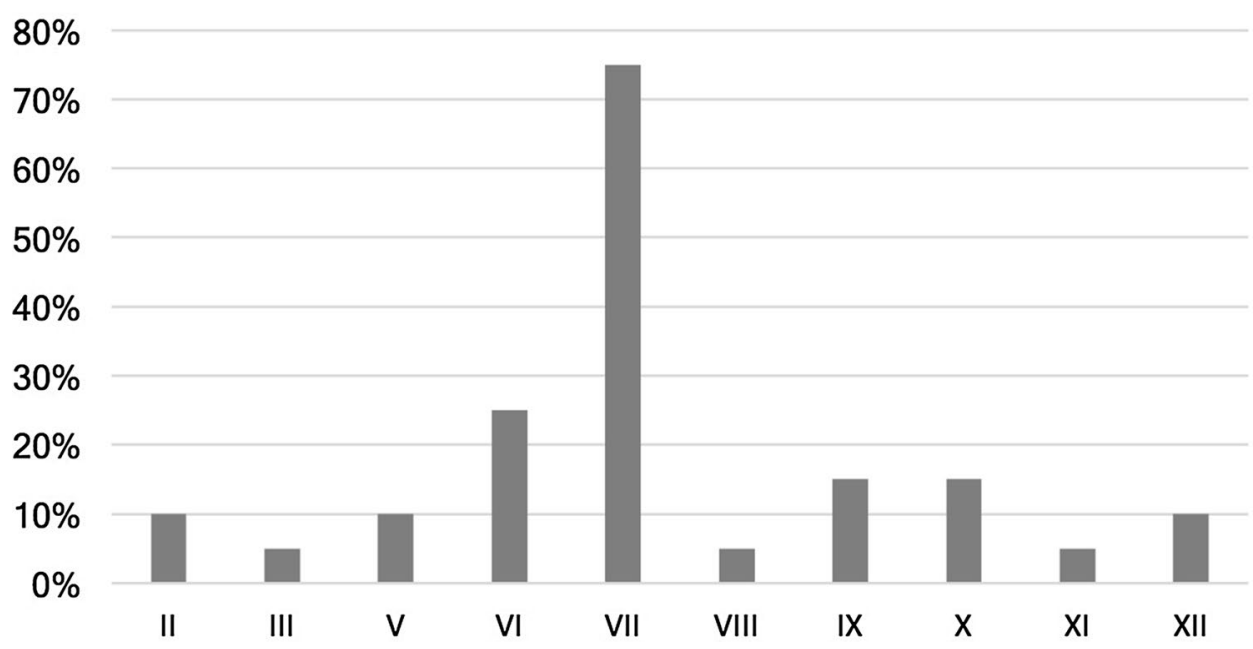


The mortality rate was $45 \%$ in 20 patients with CNP, whereas $20 \%$ in patients without CNP.

Of $46.7 \%(n=14)$ patients who survived, reports of the last clinical follow-up examination $27 \pm 34$ months after initial admission were available in eight patients. Some dysfunction of the affected nerve remained in all patients who presented with CNP on first admission $(n=4,50.0 \%)$.

\section{Discussion}

We report on outcomes of patients with skull base osteomyelitis (SBO) with special attention to the usefulness of different imaging modalities for diagnosis and during follow-up.

In accordance with the literature, the majority of patients were immunosuppressed or had diabetes [16, 18]. SBO resulted mostly from otogenic causes with typical clinical findings on admission. Otalgia and headache have been reported to be the main symptoms as confirmed in this study $[19,20]$. In some patients, the origin of the disease was not clear. Pain and mild changes in the otoscopy might occur weeks before the patient is seen by a physician or before CNP develops [19, 21, 22]. The affection of cranial nerves was high in this study. Single or multiple CNPs were present in more than two-thirds of patients on admission, predominantly the facial nerve. The presence of CNPs on admission has been stated to be a negative prognostic factor [2]. In contrast to the overall mortality rate of $36.7 \%$, almost $50 \%$ of patients with CNPs died due to SBO compared to $20 \%$ of patients without CNPs. This underlines the importance of adequate diagnosis and aggressive treatment of this disease. Lower mortality rates of $8-19 \%$ were found by other authors $[13,14,23]$. However, the rate of CNPs and/or the involvement of multiple cranial nerves was also lower compared to our results ( $8-42 \%$ versus $66.7 \%$ in the current study). On the other hand, Sokolowski et al. found CNPs in 3 out of 7 included patients who all survived [19].

One of the biggest challenges when faced with the question of SBO is to rule out malignancy. Consequently, twothirds of included patients underwent a surgical intervention such as biopsy, mastoidectomy or abscess drainage. Since long-term antibiotic therapy is necessary for SBO the most important step is isolating a pathogen. Pseudomonas aeruginosa is by far the most common pathogen and was verified in $80 \%$ of all patients in the current study. This was also observed by Lee et al. [2] and Prasad et al. [3], who found Pseudomonas aeruginosa in $74 \%$ and $80 \%$, respectively. Some authors reported that Staphylococcus aureus is another frequent causative germ $[3,13,16]$. In this study, Staphylococcus aureus was found in approximately one third but mainly in combination with other bacteria identified through microbiological swabs.
The duration of antibiotic treatment has been reported to range from four weeks to several months; in fungal SBO even up to one year [2, 11, 14, 24, 25]. In the current study cohort, broad-spectrum intravenous antibiotics were administered initially followed by culture-directed treatment with an average length of 50 days. Increasing rates of ciprofloxacin-resistant Pseudomonas aeruginosa with no influence on mortality and morbidity have been reported [24]. In this study, 3-MDRGN Pseudomonas aeruginosa appeared in only two patients who were admitted within the last two years of the study period. As this number will probably increase in the future, attention should be paid to the correct choice of antibiotic agent.

Early discontinuation of treatment can lead to disease relapse; among improvement of symptoms, the duration of treatment depends on follow-up imaging. Which imaging modality is most useful to assess treatment efficacy is still a matter of discussion since conventional methods do not properly represent disease activity. At least one CT scan was performed in all patients of the current study. The most common radiological findings were soft tissue changes and bone erosion in approximately $50 \%$ of patients. Follow-up scans revealed similar results compared to first scans and failed to reveal disease progression or regression in up to $80 \%$ after four to nine months. Within the first two months after the initial CT scan, the highest progression rate was found. Nevertheless, CT is the most commonly used imaging method to diagnose and monitor SBO [7], as also underlined in this study. There is no doubt, that the assessment of bone erosion and demineralization can be shown best on CT imaging. However, initial findings in SBO can be non-specific and bone remineralization occurs late after disease resolution $[22,26]$. Consequently, this modality seems less reliable to monitor treatment response.

Interestingly, an additional MRI was not performed on every patient of the present study cohort. A second MRI scan was performed in $40 \%$ after 2.7 months on average. The second scan revealed disease progression in $50 \%$, whereas one out of four showed regression or no change compared to the first scan. Follow-up MRI scans revealed a greater proportion of disease progression compared to CT scans. Keeping the radiation burden in mind, MRI seems to be more suitable compared to CT for follow-up. However, an abnormal bone marrow signal on MRI can be persistent for a up to 12 months after successful treatment [27]. In order to overcome the drawbacks of conventional radiologic examinations in SBO, nuclear medicine imaging methods can aid the diagnosis and monitoring of therapy [9]. Only 15 nuclear medicine imaging examinations were performed in a third of the included patients. Due to reduced availability, examinations were performed after standard imaging methods were carried out and an enhanced tracer uptake was found in almost all cases. Unfortunately, no follow-up 
examination was performed on a regular basis so that we cannot compare the results properly. Tc-99m-MDP was the most frequently used tracer in bone scintigraphy and was combined with SPECT in most cases. Even a $10 \%$ rise in osteoblastic activity can be detected with this tracer and is therefore useful in the beginning of the disease [28, 29]. In contrast, Tc-99 uptake is also increased in post-operative and malignant conditions and can still be present in disease resolution [26]. Therefore, it is less suitable for follow-up. Out of the four Tc-99 labelled leucocyte scans, two were found to be normal although clear deterioration was found on conventional imaging modalities. Besides the inability to detect low-grade infectious foci, Tc-99 labelled leucocytes are more expensive and the diagnostic value is questionable. Beta-emitting tracers like FDG enable the detection of ongoing neutrophil activity in metabolically active tissue and therefore, can detect inflammation. FDG has been recommended as first choice in diagnosis and for decisionmaking when to stop treatment $[9,30]$. In the present study, FDG was used in PET-CT or PET-MRI in only three cases. One patient had recurrent disease and underwent PETCT 6 months after the first admission, which resulted in the continuation of intravenous antibiotics for another four weeks. Unfortunately, this patient had his fourth relapse nine months after first admission and died due to progressive disease. Two other patients were also monitored with PET-CT or PET-MRI and treatment was continued because of increased tracer-uptake. Nowadays, the availability of hybrid techniques such as PET-MRI is poor but there is good potential for disease assessment and treatment monitoring in the future.

\section{Conclusion}

Skull base osteomyelitis (SBO) is a life-threatening disease with a high complication rate including persistent cranial nerve palsy and death. Unspecific headache or otalgia, even with normal findings in otoscopy, should raise the possibility of SBO in patients with diabetes or immunosuppression. Early microbiologic swabs are crucial to identify the pathogen before antibiotic treatment is administered. As no single imaging method is able to assess the full extent of SBO, a combination of anatomical and nuclear medicine imaging methods should be considered for diagnosis. Based on our data, follow-up imaging should be performed not as early as two months after the initial scan if there is no clinical evidence for disease progression. MRI should be the preferred modality rather than CT. Disease monitoring and consequently the time when treatment can be stopped is probably best performed by means of functional imaging.
Author contributions All authors have approved the final manuscript.

Funding Open Access funding provided by Medical University of Vienna.

\section{Compliance with ethical standards}

Conflict of Interest The authors declare no conflicts of interest.

Open Access This article is licensed under a Creative Commons Attribution 4.0 International License, which permits use, sharing, adaptation, distribution and reproduction in any medium or format, as long as you give appropriate credit to the original author(s) and the source, provide a link to the Creative Commons licence, and indicate if changes were made. The images or other third party material in this article are included in the article's Creative Commons licence, unless indicated otherwise in a credit line to the material. If material is not included in the article's Creative Commons licence and your intended use is not permitted by statutory regulation or exceeds the permitted use, you will need to obtain permission directly from the copyright holder. To view a copy of this licence, visit http://creativecommons.org/licenses/by/4.0/.

\section{References}

1. Chang PC, Fischbein NJ, Holliday RA (2003) Central skull base osteomyelitis in patients without otitis externa: imaging findings. AJNR Am J Neuroradiol 24:1310-1316

2. Lee S, Hooper R, Fuller A et al (2008) Otogenic cranial base osteomyelitis: a proposed prognosis-based system for disease classification. Otol Neurotol 29:666-672. https://doi.org/10.1097/ MAO.0b013e318179972f

3. Prasad SC, Prasad KC, Kumar A et al (2014) Osteomyelitis of the temporal bone: terminology, diagnosis, and management. J Neurol Surg B Skull Base 75:324-331. https://doi. org/10.1055/s-0034-1372468

4. Ducic Y (2003) Management of osteomyelitis of the anterior skull base and craniovertebral junction. Otolaryngol Head Neck Surg 128:39-42. https://doi.org/10.1067/mhn.2003.9

5. Rothholtz VS, Lee AD, Shamloo B et al (2008) Skull base osteomyelitis: the effect of comorbid disease on hospitalization. Laryngoscope 118:1917-1924. https://doi.org/10.1097/MLG.0b013 e31817fae0d

6. Chawdhary G, Liow N, Democratis J, Whiteside O (2015) Necrotising (malignant) otitis externa in the UK: a growing problem. Review of five cases and analysis of national Hospital Episode Statistics trends. J Laryngol Otol 129:600-603. https://doi. org/10.1017/S002221511500105X

7. Chawdhary G, Pankhania M, Douglas S, Bottrill I (2017) Current management of necrotising otitis externa in the UK: survey of 221 UK otolaryngologists. Acta Otolaryngol 137:818-822. https://doi. org/10.1080/00016489.2017.1295468

8. Balakrishnan R, Dalakoti P, Nayak DR et al (2019) Efficacy of HRCT imaging vs SPECT/CT scans in the staging of malignant external otitis. Otolaryngol Head Neck Surg 161:336-342. https ://doi.org/10.1177/0194599819838834

9. van Kroonenburgh AMJL, van der Meer WL, Bothof RJP et al (2018) Advanced imaging techniques in skull base osteomyelitis due to malignant otitis externa. Curr Radiol Rep 6:3-14. https:// doi.org/10.1007/s40134-018-0263-y

10. Chen Y-H, Hsieh H-J (2013) Single photon emission computed tomography/computed tomography for malignant otitis externa: 
lesion not shown on planar image. Am J Otolaryngol 34:169-171. https://doi.org/10.1016/j.amjoto.2012.11.001

11. Ridder GJ, Breunig C, Kaminsky J, Pfeiffer J (2015) Central skull base osteomyelitis: new insights and implications for diagnosis and treatment. Eur Arch Otorhinolaryngol 272:1269-1276. https ://doi.org/10.1007/s00405-014-3390-y

12. Rubin J, Yu VL (1988) Malignant external otitis: insights into pathogenesis, clinical manifestations, diagnosis, and therapy. Am J Med 85:391-398. https://doi.org/10.1016/0002-9343(88)90592 $-\mathrm{x}$

13. Chen C-N, Chen Y-S, Yeh T-H et al (2010) Outcomes of malignant external otitis: survival vs mortality. Acta Otolaryngol 130:89-94. https://doi.org/10.3109/00016480902971247

14. Spielmann PM, Yu R, Neeff M (2013) Skull base osteomyelitis: current microbiology and management. J Laryngol Otol 127(Suppl 1):S8-12. https://doi.org/10.1017/S0022215112002356

15. Chandler JR (1968) Malignant external otitis. Laryngoscope 78:1257-1294. https://doi.org/10.1288/00005537-19680 8000-00002

16. Johnson AK, Batra PS (2014) Central skull base osteomyelitis: an emerging clinical entity. Laryngoscope 124:1083-1087. https:// doi.org/10.1002/lary.24440

17. Sandner A, Henze D, Neumann K, Kösling S (2009) Value of hyperbaric oxygen in the treatment of advanced skull base osteomyelitis. Laryngorhinootologie 88:641-646. https://doi. org/10.1055/s-0029-1214394

18. Sreepada GS, Kwartler JA (2003) Skull base osteomyelitis secondary to malignant otitis externa. Curr Opin Otolaryngol Head Neck Surg 11:316-323. https://doi.org/10.1097/00020840-20031 0000-00002

19. Sokołowski J, Lachowska M, Karchier E et al (2019) Skull base osteomyelitis: factors implicating clinical outcome. Acta Neurol Belg 119:431-437. https://doi.org/10.1007/s13760-019-01110-w

20. Das S, Iyadurai R, Gunasekaran K et al (2019) Clinical characteristics and complications of skull base osteomyelitis: a 12-year study in a teaching hospital in South India. J Family Med Prim Care 8:834-839. https://doi.org/10.4103/jfmpc.jfmpc_62_19

21. Patmore H, Jebreel A, Uppal S et al (2010) Skull base infection presenting with multiple lower cranial nerve palsies. Am J Otolaryngol 31:376-380. https://doi.org/10.1016/j.amjoto.2009.04.001

22. Clark MPA, Pretorius PM, Byren I, Milford CA (2009) Central or atypical skull base osteomyelitis: diagnosis and treatment. Skull Base 19:247-254. https://doi.org/10.1055/s-0028-1115325
23. Glikson E, Sagiv D, Wolf M, Shapira Y (2017) Necrotizing otitis externa: diagnosis, treatment, and outcome in a case series. Diagn Microbiol Infect Dis 87:74-78. https://doi.org/10.1016/j.diagm icrobio.2016.10.017

24. Berenholz L, Katzenell U, Harell M (2002) Evolving resistant pseudomonas to ciprofloxacin in malignant otitis externa. Laryngoscope 112:1619-1622. https://doi.org/10.1097/00005537200209000-00017

25. Djalilian HR, Shamloo B, Thakkar KH, Najme-Rahim M (2006) Treatment of culture-negative skull base osteomyelitis. Otol Neurotol 27:250-255. https://doi.org/10.1097/01.mao.0000181185 .26410 .80

26. Adams A, Offiah C (2012) Central skull base osteomyelitis as a complication of necrotizing otitis externa: imaging findings, complications, and challenges of diagnosis. Clin Radiol 67:e7-e16. https://doi.org/10.1016/j.crad.2012.02.004

27. Karantanas AH, Karantzas G, Katsiva V et al (2003) CT and MRI in malignant external otitis: a report of four cases. Comput Med Imaging Graph 27:27-34. https://doi.org/10.1016/s0895 -6111(02)00052-6

28. Seabold JE, Simonson TM, Weber PC et al (1995) Cranial osteomyelitis: diagnosis and follow-up with In-111 white blood cell and Tc-99m methylene diphosphonate bone SPECT, CT, and MR imaging. Radiology 196:779-788. https://doi.org/10.1148/radio logy.196.3.7644643

29. Asimakopoulos P, Supriya M, Kealey S, Vernham GA (2013) A case-based discussion on a patient with non-otogenic fungal skull base osteomyelitis: pitfalls in diagnosis. J Laryngol Otol 127:817-821. https://doi.org/10.1017/S002221511300145X

30. Stern Shavit S, Bernstine H, Sopov V et al (2019) FDG-PET/ $\mathrm{CT}$ for diagnosis and follow-up of necrotizing (malignant) external otitis. Laryngoscope 129:961-966. https://doi.org/10.1002/ lary. 27526

Publisher's Note Springer Nature remains neutral with regard to jurisdictional claims in published maps and institutional affiliations. 